\title{
Multi-scale simulation of ground support designs for extreme tunnel closure
}

\author{
D.A. Beck Beck Arndt Engineering Pty Ltd, Australia
}

S. Kassbohm Beck Arndt Engineering Pty Ltd, Australia

G. Putzar Beck Arndt Engineering Pty Ltd, Australia

\begin{abstract}
The numerical simulation of ground support requires that the ground deformation and support response are both captured accurately. The mechanisms of rock mass damage, dilation and deterioration must first be simulated sufficiently to produce realistic tunnel deformation in $3 D$, then the physical response of the support elements must be realistic if the support and loading systems will come to a realistic equilibrium.

This requires the simulation of rock mass and mechanical behaviour on a number of different length scales and demands a level of similitude that is not commonly implemented for mine models. The load-displacement response of both the support and the rock mass around excavations must be correct for the models to be valid.

A multi-scale approach to mine deformation modelling can be used to improve the similitude of capacity and demand simulation of ground support. Case studies are used to demonstrate the behaviour of several heavy support systems using this approach. Some proposed support designs for a deep cave subject to high stress and expected significant drive closure are tested and the limitations and vulnerabilities of the support systems are exposed.
\end{abstract}

The modelling methods used to simulate the mine deformation, drive behaviour and support system response are discussed and some sufficiency requirements for similar analysis are highlighted.

\section{Introduction}

Ground support and reinforcement is installed in a strain free, or specified load condition into a deformed, discontinuous rock mass. The subsequent additional loads that develop in the support system are a function of additional displacements in the rock mass and the resultant forces generated by blocks and wedges that would otherwise be kinematically free to fall or slide.

To evaluate the performance of a support system, analysis must sufficiently capture the evolution and timing of the discontinuous deformation within the rock mass. The support systems also interact with the rock in a complex way and the support system itself is a complex, 3D assembly of elements interconnected by other elements or the rock mass. The analysis of a rock-support-reinforcement system is further complicated if the support is to be simulated beyond the point of failure of some of the elements as the problem becomes significantly non-linear; the failure of one part of the system could lead to failure of another and another and so on.

Put simply, the simulation of ground support is a complex problem demanding the highest level of realism. If the simulated 'loading system' displacements or the simulated support system load-displacement response is incorrect, the evaluation will not be sufficient.

\section{Displacement realistic modelling}

Apart from the quality of input data for model variables (material properties, boundary conditions etc), there are several model fundamentals that limit the ability of a model to simulate realistic displacements. These things are critical to sufficient analysis, as even if the model variables were otherwise correct, the model would be incapable of simulating deformation realistically. 
The main categories of these fundamental model characteristics are: dimensionality, geometry, constitutive behaviour and scale; these categories are very broad and cover most decisions made when developing a model. It is beyond the scope of this paper to cover all of these aspects in detail, but in the mining context it is possible to specify some broadly applicable requirements for sufficient displacement realistic modelling:

- Generally, displacement realistic models will usually be 3D. A specific situation in a specific location may be able to be approximated using a 2D model for some stage of the life of an excavation, but when estimating the demand that a system will be subjected to, the reasonable range of outcomes that need to be considered will span too many situations in which the complete 3D stress path, including the intermediate stages of the stress path, is critical to the outcome. Even monolithic forms such as universal beam sets discussed in more detail below, most often fail via a mechanism cannot to be simplified to a $2 \mathrm{D}$ problem.

- Almost all deformation problems require a 3D model to properly capture geometric effects. Capturing the geometry sufficiently in 3D is also essential to simulate the stress path. This means not only representing the shape of excavations and structure, but also simulating the timing and the excavation process. The process of forming the geometry - that is the intermediate geometries formed during the excavation process - will shape the extent and magnitude of damage and the deformation field. If the intermediate excavation stages are too far apart in a model, the effects cannot usually be understood or quantified using experience alone.

- The effective constitutive response of a model is a function of the governing physics that a model can capture, and accounts for the constitutive material model that is adopted, the element type and density or any other inherent characteristic that limits or constrains the behaviour of the simulated material. Assuming a sufficient type and density of elements is used and the analytical framework can solve for the physical response that needs to be captured, then the practical challenge for ground support problems is to simulate the continuous and discontinuous parts of the deformation field around the excavation being considered.

This implies that the model will capture the stress strain behaviour well enough to model the extent and magnitude of damage around the excavation (for example, with a strain-softening, dilatant, large strain model), but also incorporate sufficiently small scale structure so that the discontinuous deformation and kinematics of the problem are captured.

- Different length scales are interconnected; the stability and deformation at larger scales drives deformation at smaller scales. The interconnectedness also works in the opposite direction; small scale rock mass behaviour is the nucleus of stability of larger parts of the mine that sum to affect global scale deformation. In practice, the length scales are not discrete and there are no distinct boundaries, it is simply helpful to explain the concept of scale in a mine problem in this way.

Multi-scale modelling is any modelling where there is defined precision for deformation and distortion of the rock mass at multiple length scales, and this requires that excavations and discontinuities are represented across the length scales (or at least sufficiently bracketing the length scale of interest). Generally, the behaviour at each length scale is built upon to simulate successively larger length scales. This could be achieved by incorporating geometry and sequencing details at a resolution and complexity suiting the smallest length scale of interest in the problem, inside a model with similar geometric complexity at longer length scales or using appropriate sub modelling techniques (see Beck, 2008; Beck and Reusch, 2009).

\section{An example procedure}

At this time, it is not efficient to build a single mine-scale model with all scales of necessary structure from drive scale to mine scale. Instead, a multi-scale sub modelling approach can be adopted, using a larger scale models to provide boundary displacements for smaller scale models, each with successively smaller scale details. All scales of model have different purposes and incorporate different details:

- The purpose of the donor models is not to replicate the smaller scale displacements; it is to produce sufficiently correct displacements to drive the boundary of the smaller scale models, i.e. the 'sub' models. 
- The representative elementary volume (REV or RVE) is used as a guide to the length scale below which structures will not be represented explicitly. A procedure for identifying the REV is outlined in Beck and Reusch, 2009. For most problems (not all), the REV in the donor model is the smallest volume needed to homogenise the effects of joint sets on rock mass scale properties. Heterogeneities larger than joints, such as faults or shears may need to be considered explicitly.

- The support scale sub model has the purpose of producing displacements at a wall, or support element scale, including the nature and magnitude of the discontinuous deformation around the tunnel and at the tunnel surface. This means that smaller structures that can dislocate in the area of influence of the tunnel need to be explicitly represented.

A simple example of donor and sub models is shown in Figure 1. In this case donor models of a mine scale and 1/4 mine scale were used in series to produce the support model boundary displacements and the support elements are included explicitly in the sub model. Each scale of model is a strain softening, dilatant, discontinuum FE model utilising higher order elements. Over 100 extraction steps were used in the donor models and at each step the displacements were transferred to corresponding nodes of the smaller model. Fewer steps were needed in the support scale model owing to the late extraction of the drive (pre-mining displacements could be simplified). The level of detail in support model included cut by cut extraction, installation of support at the correct timing in the sequence and an explicit representation of the bolts, fibrecrete and steel mesh.

A more complex example and a different approach is shown in Figure 2 for a panel cave. The purpose of this model was to evaluate the capacities of the steel arches and steel sets for drawpoint support. A global model of the entire mine was used to drive the boundaries of a 1/10 footprint sub model spanning from below the extraction level to above the undercut, but because many support designs needed to be tested, the drives within the $1 / 10$ footprint sub model were only filled with supporting 'filler' elements approximating the pre-failure stiffness of the systems that were proposed. The displacements at the drive surface could then be applied to the explicit models of the support elements or directly compared to published load-displacement data. In a more detailed study the support could be included directly in the 1/10 footprint model as this model has the required resolution of discontinuities and the capacity; the simplification was only for efficiency.

In this particular example, the $1 / 10$ footprint models incorporate discrete fracture networks (DFNs) to represent drive scale structure. The included discontinuities are shown in the sub model plots in Figure 2. The DFNs are based on a simple statistical representation of the discontinuity spatial distribution. The procedure for developing these FE DFN models was as follows:

- scan line mapping recorded the dip, dip direction, trace length and set number of discontinuities in several locations

- scan lines were de-surveyed to establish the distribution of spacings and persistence along typical drives

- DFNs were constructed that match these distributions and applied to FE models of as-built excavations as shown in Figure 2.

The FE DFN models were inspected to ensure that the same tunnel scale discontinuity distribution was achieved in the model, and the persistence of the joints was adjusted iteratively to achieve a best qualitative match. Note: only tunnel scale or larger discontinuities were included; smaller scale (i.e. $~<1 \mathrm{~m}$ persistence) discontinuities were homogenised.

An issue that arises is that the global scale models in both cases represent geotechnical domains using rock mass scale properties (the joints are smeared into a continuum representation for each rock type), whereas the FE DFN models contains the smeared joints. This means that different material properties are needed for the DFN models than for the global models. Generally, as the global scale model would be calibrated, or else material properties at that scale already determined and agreed upon, the joint and continuum mass properties in the DFN can be iteratively adjusted until the response at the REV scale is the same in both scales of models. 


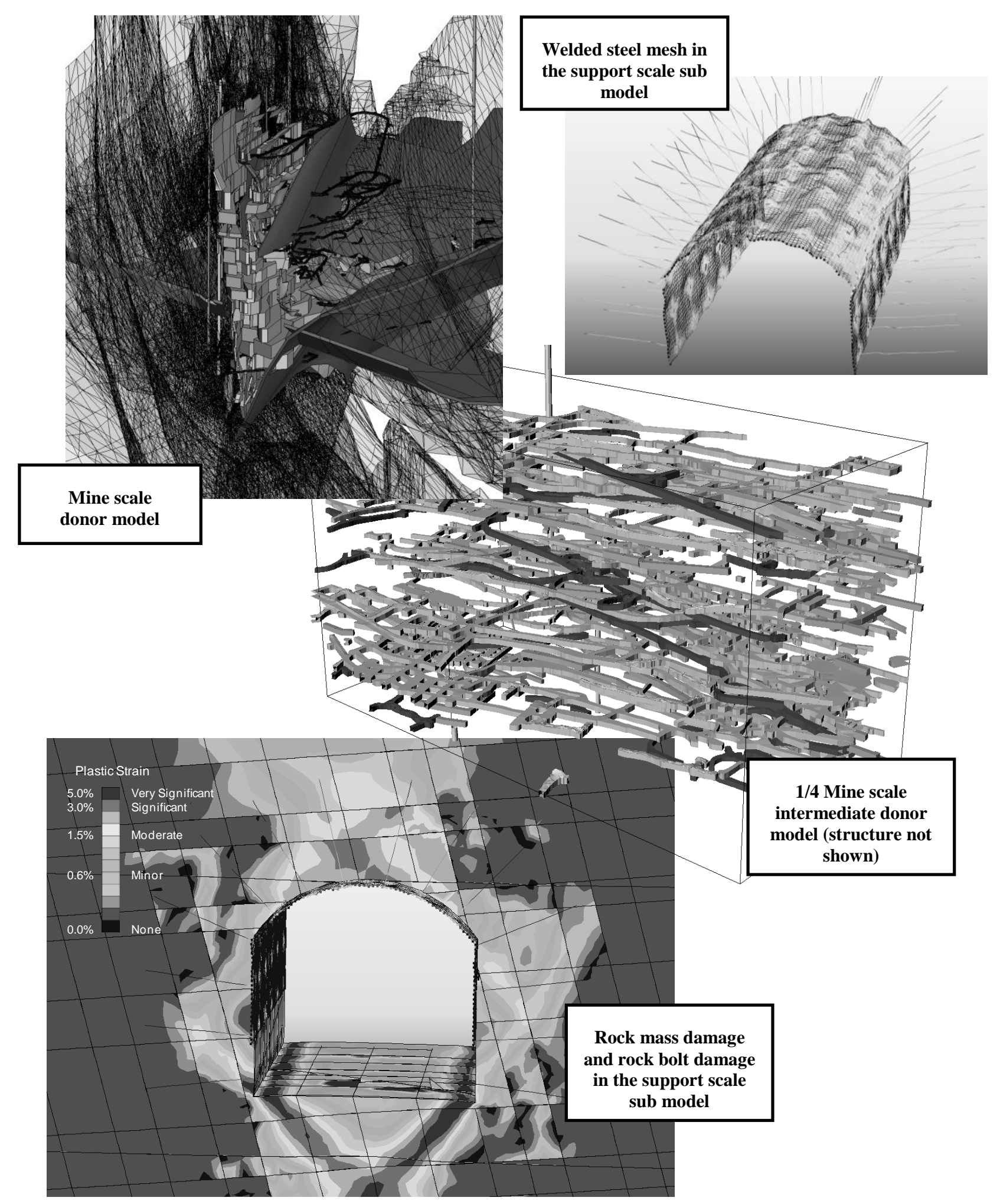

Figure 1 Example of 1/4 mine scale model complexity as built and with an example of simulated support load in development 


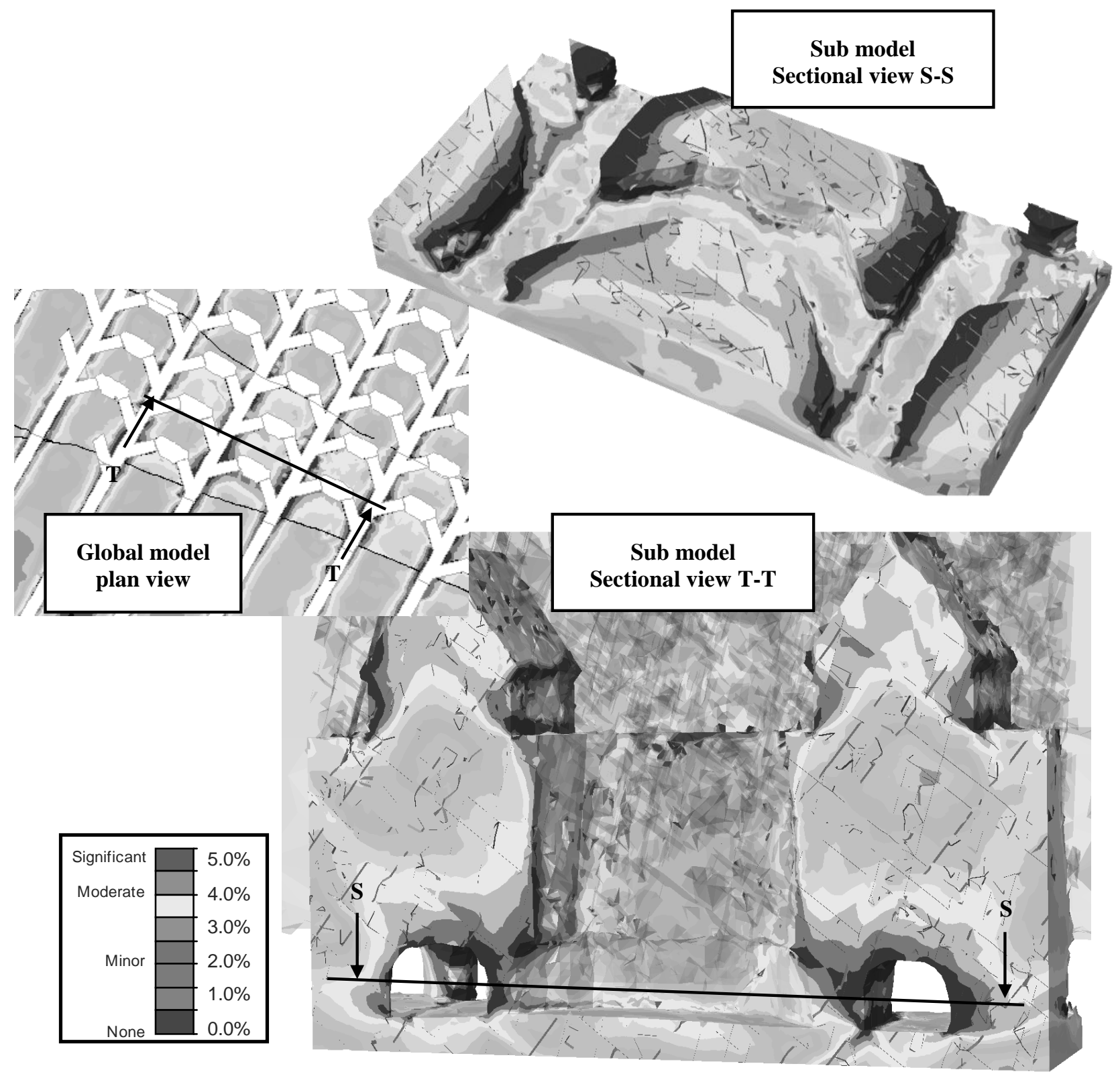

Figure 2 Example sections showing plastic strain, through a 1/10 footprint DFN and global donor model used to produce drive surface displacements to test steel arch and steel set support. Only part of the model is shown

The results for excavation closure from this model at an isolated location of high drive closure are plotted in Figure 3. This shows representative, underlying inwards wall movement versus model step in the 1/10 footprint sub model at three key locations in the sub model; a drawpoint, crosscut and drawpoint intersection. Note that the specific location was selected on the basis of expected large deformation and that the model is calibrated using measured drive closure. Almost all of the rest of the footprint shows much lower expected drive closure. The model indicates that at this selected location, with effective TH or universal beam support, up to $275-300 \mathrm{~mm}$ of closure is eventually expected after propagation of the cave.

For this example, two candidate support designs were considered in detail: TH Arches (WASM, pers. comm., 2007) and steel ('I', or universal beam) sets. In the numerical tests of these support designs, the final loads generated by donor model can be applied as simulated, or amplified until the support systems fail to assess the ultimate capacity of the support systems. 


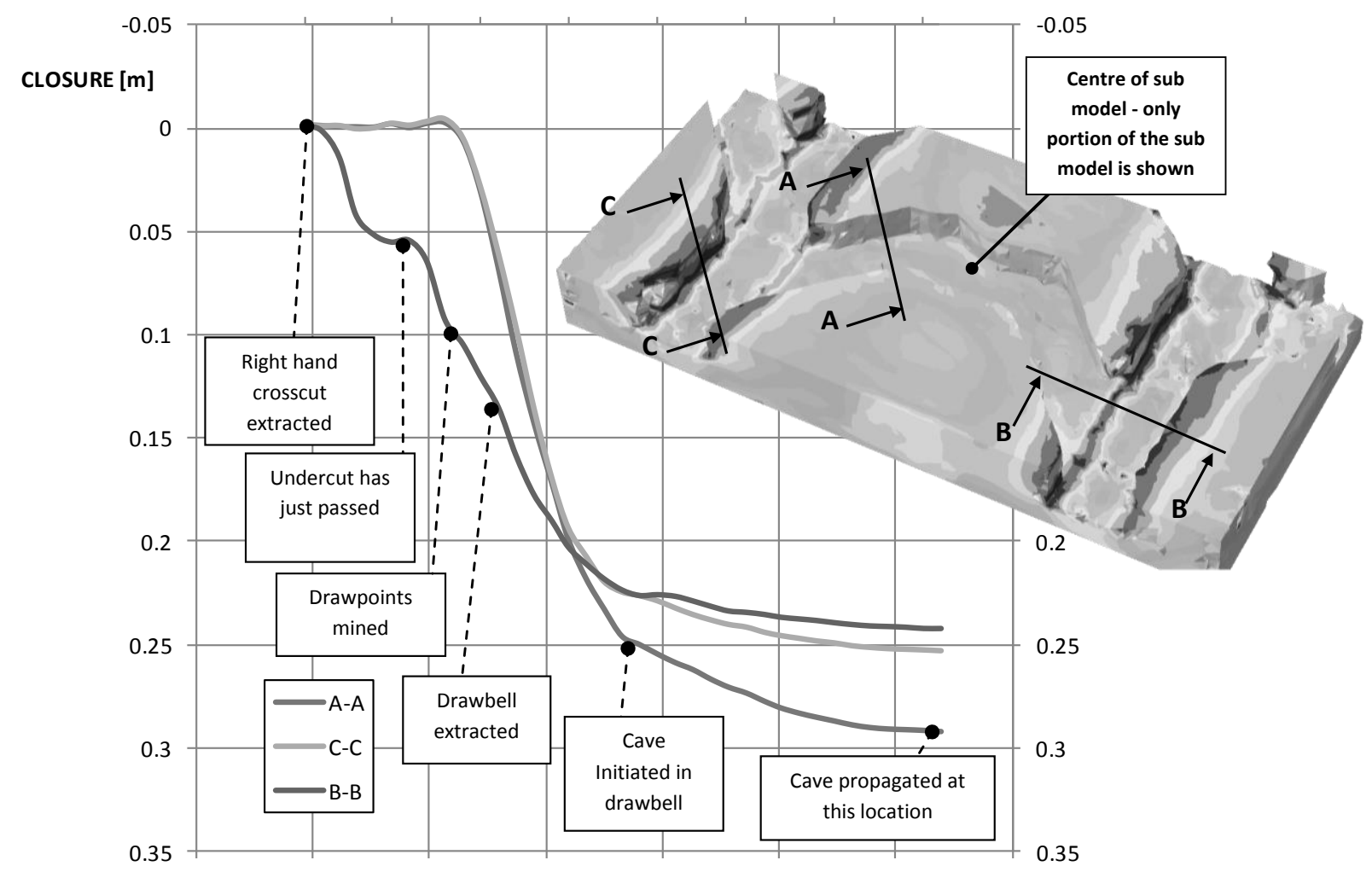

Figure 3 Simulated closure at selected locations in the example front cave

A TH arch design is shown in Figure 4. It is a high capacity design specified for extreme drive closure. Features of the design include embedded 'crush' elements in the fibrecrete and mesh over the TH arches and fibrecrete. The intent of the design is to provide maximum support pressure over the largest range of drive closure. The capacity simulation for this support is presented in Figure 4 at 200-300 mm of closure and $300-400 \mathrm{~mm}$ of closure respectively, in this case using drive deformation information from another mine planning to utilise circular tunnels to assist in managing significant ground movement. The results show that at $200-300 \mathrm{~mm}$ of closure the crush elements show significant strain and are effectively protecting the surface support as required. By 300-400 $\mathrm{mm}$ of closure, the TH arches begin to buckle and capacity is lost. This represents the ultimate theoretical capacity of the system in the example mine's application, even though drive instability will not necessarily occur at this level of closure. In practice, irregularities in the drive shape could also promote higher fibrecrete damage much earlier than this so much lower fibrecrete capacity should be expected. If the crush elements are not installed, fibrecrete capacity would also be a fraction of that estimated here.

Universal beam sets are a more common support design for draw points. Draw points must manage the competing needs of being heavily deformed, as well resisting wear and impacts. It is also often asserted that the shape of the draw point has a defining impact on flow within the cave, so a 'square' brow is often specified.

The most problematic constraint is the asymmetric deformation that results from the shape of the draw point pillars (typically diamond shaped) which skews the drive along the axis, especially if the pillar core is damaged as shown in Figure 5 from a model of a heavily deformed draw point from another example mine. While the total closure is similar to that experienced in the adjacent crosscuts, the nature of the deformation can problematic for stiff monolithic support. 


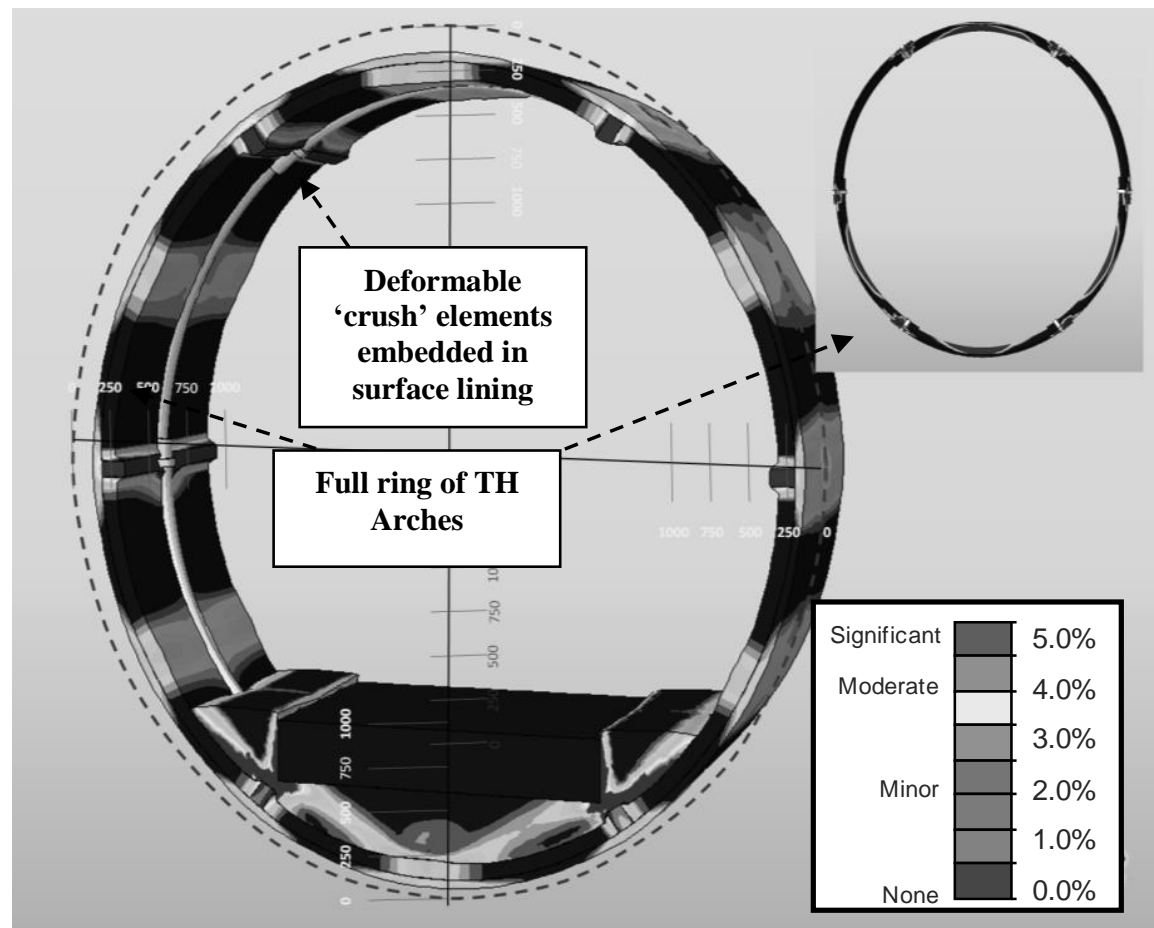

(i) 200-300 $\mathrm{mm}$ of closure

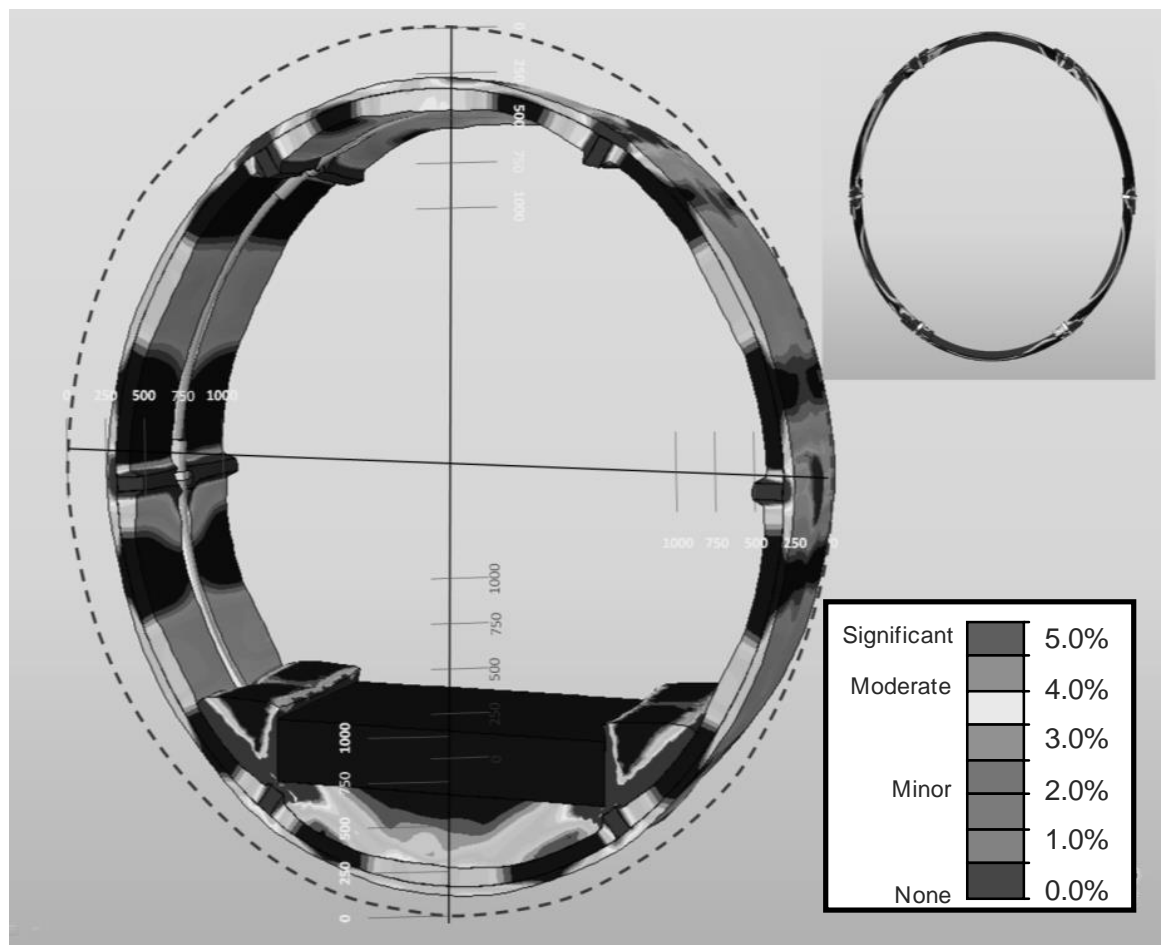

(ii) $300-400 \mathrm{~mm}$ of closure

Figure 4 Simulated shear zone support damage (plastic strain), with 80 MPa roadway 
A common steel set design for block caves is shown in Figure 6. Initially, as the support is installed at passive loads, significant ground movement is required for any substantial load to be taken up. Then, there is a large disparity in stiffness between the areas of drive affected by the sets and the spans in between. This effect can be seen in Figure 7(i) which shows the pattern of radial pressure between the wall of the drive and the support system. The support pressures generated are much higher adjacent to the sets than they are in the spans between, suggesting closer spacing is needed for at least this application. The figure also shows the pressure generated by the sets compared to the deformation at selected locations around the square sets (Figure 7(ii)) i.e. at the shoulder and grade line, immediately adjacent to one of the sets. The sets 'point load' resulting in massive pressures at certain points. It must be noted that the 'point loads' are not representative of the average support pressure. Generally this would be an average of the loads shown in the figure, probably closer to $1 / 5-1 / 6$ of the indicated point loads.

The simulated performance of this system is shown in Figure 8 at $325 \mathrm{~mm}$ of closure. Between 250 and $325 \mathrm{~mm}$ of closure at the brow, the model showed the single steel sets start to buckle (see Figure 8, top right), but there is significant concrete damage in areas including the apron along the footings of the arch before this. The twin sets at the brow perform better than the single sets, showing no signs of buckling. The type of buckling in this case is driven partly by the mode of drive deformation shown in Figure 5 and anecdotally this torsional failure at the shoulder is a common mechanism for steel set damage. An example comparing set damage from one mine and a close view of the modelled buckling is shown in Figure 9.

Based on the analysis, for the example mines environment it was concluded that as a result of the inherent nature of the support design, the drive may at least require major repair or set replacement beyond $325 \mathrm{~mm}$ of closure, neglecting effects due to wear or oversize handling.

Another use for the model is to better understand the mechanism for the high levels of damage. Results for rock mass damage - interpreted using plastic strain — through the drawpoint pillars, minor apex and in the walls and back at selected sections of the sub-model excavations are shown in Figure 10: a) minor to moderate pillar core; b) significant pillar core damage, at two different stages of extraction.

The eventual significant closure is correlated with the loss of pillar core stability. Interpreting pillar core conditions using plastic strain is consistent with standard geotechnical practice. Conventional theorems of plastic collapse for limit analysis are well documented (Hodge, 1958; Davis, 1968; Lubliner, 1990; Drucker et al., 1952; Hill, 1951).

The detailed modelling correlates drive closure and pillar core stability for the example application, and although the actual level of closure compared to the pillar damage is specific to the rock type and configuration of the excavations, the correlation between stability generally and with levels of plastic strain should be more transferrable:

- Pillar cores with significant damage should be interpreted as failed. The adjacent excavations will also be deformed.

- From a support perspective these drives are candidates for heavy support and planned rehabilitation.

- Pillar cores with moderate damage should be marginal - some will be interpreted as failed. In these pillars degradation has increased but the actual deformation is still only moderate. The adjacent drives should not be significantly deformed, though additional load will lead to increased damage and deformation in the pillar and adjacent excavations.

- These drives are also candidates for heavy support, although many will not fail, or may no longer be required by the time significant deformation occurs. The support might only be considered following an assessment of the risks and circumstances in each location.

- Pillar cores with minor damage should be expected to be stable. Calibration suggests that at this level of damage, the rock mass is commencing to yield but a significant degradation in strength has not occurred. Also, as the deformation is low, deformation in adjacent excavations will also be low and heavy support is not required. 


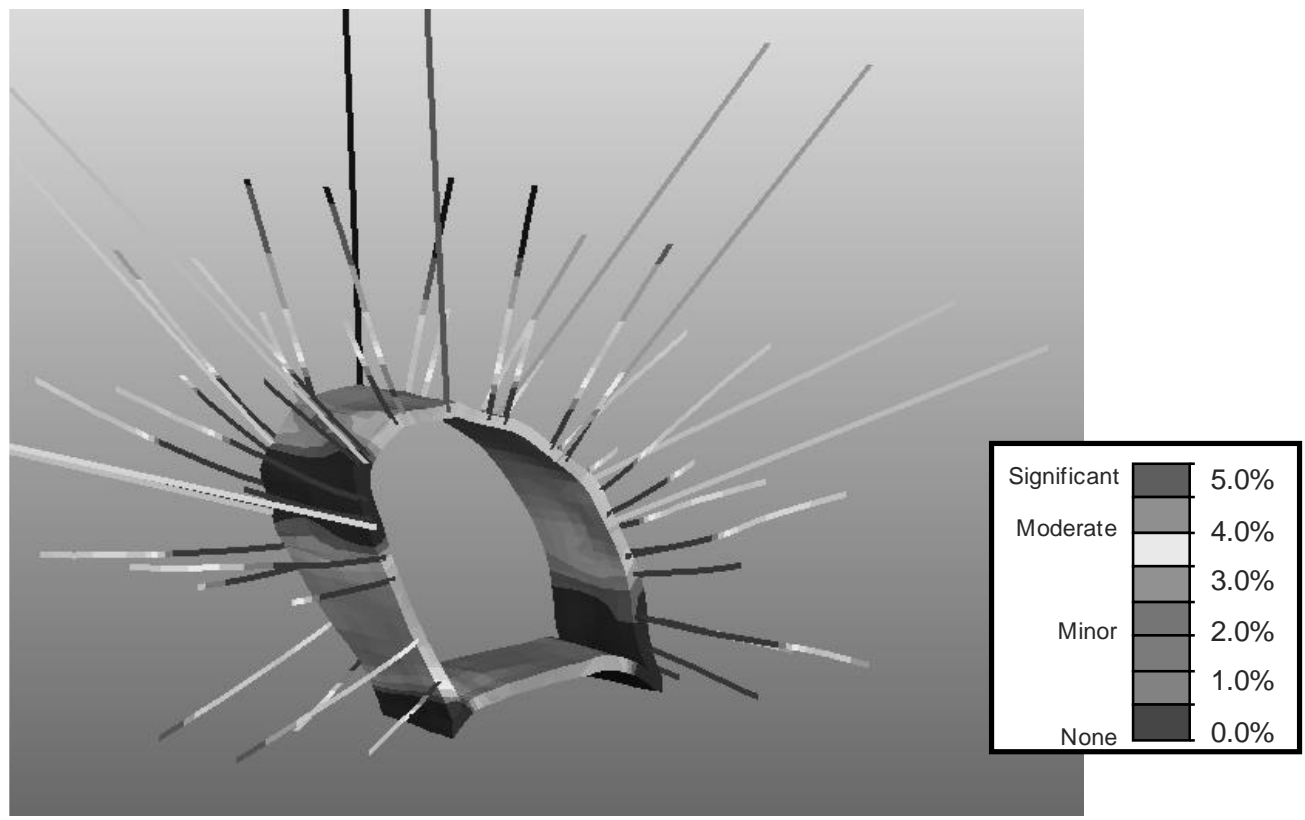

Figure 5 Exaggerated draw point deformation, showing severely asymmetric distortion of the drive caused by geometry of the draw point pillars and approximate simulated drawpoint closure

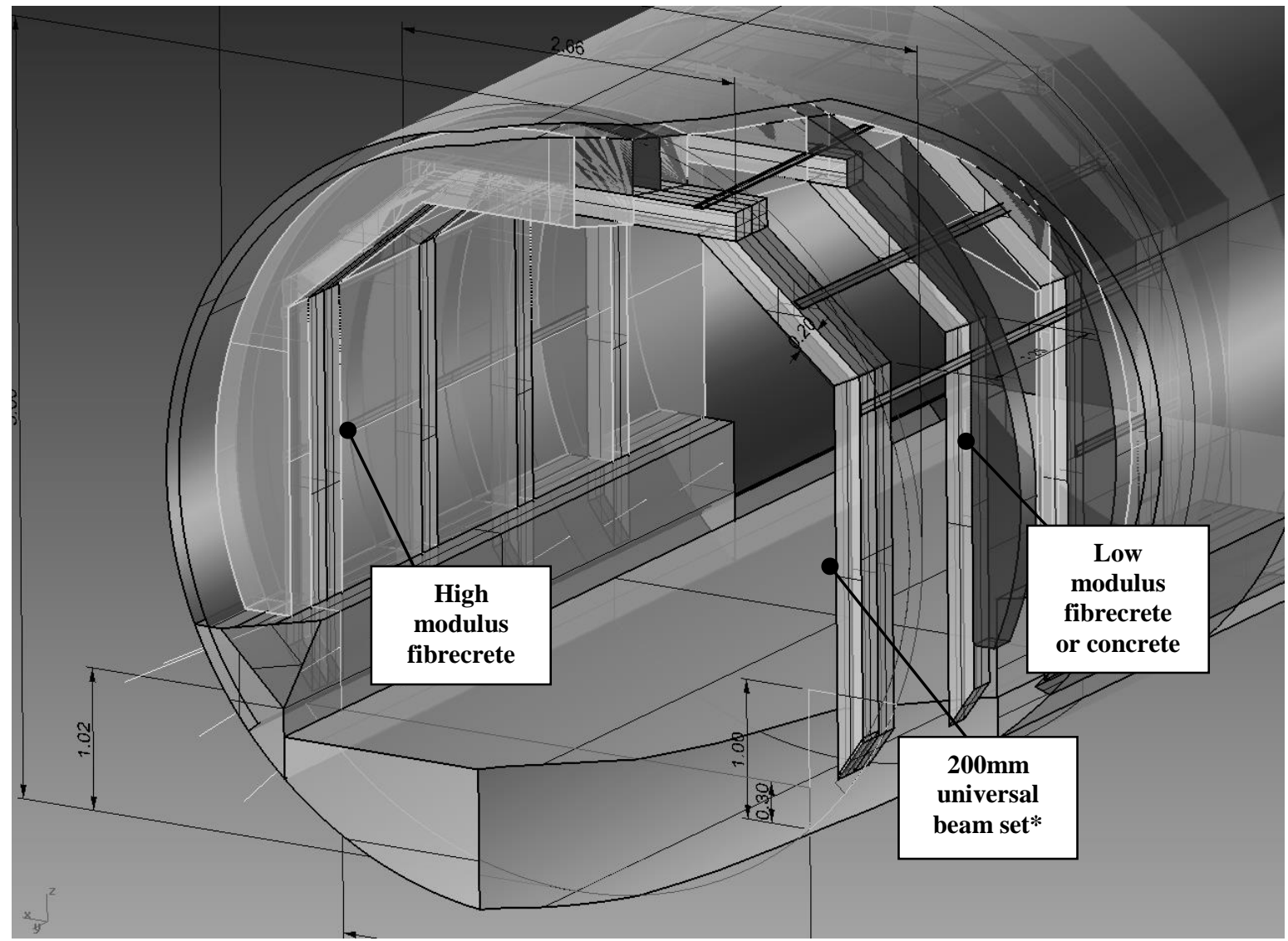

* Twin sets at brow, single sets elsewhere

Figure 6 A common steel set design for block cave drawpoints 


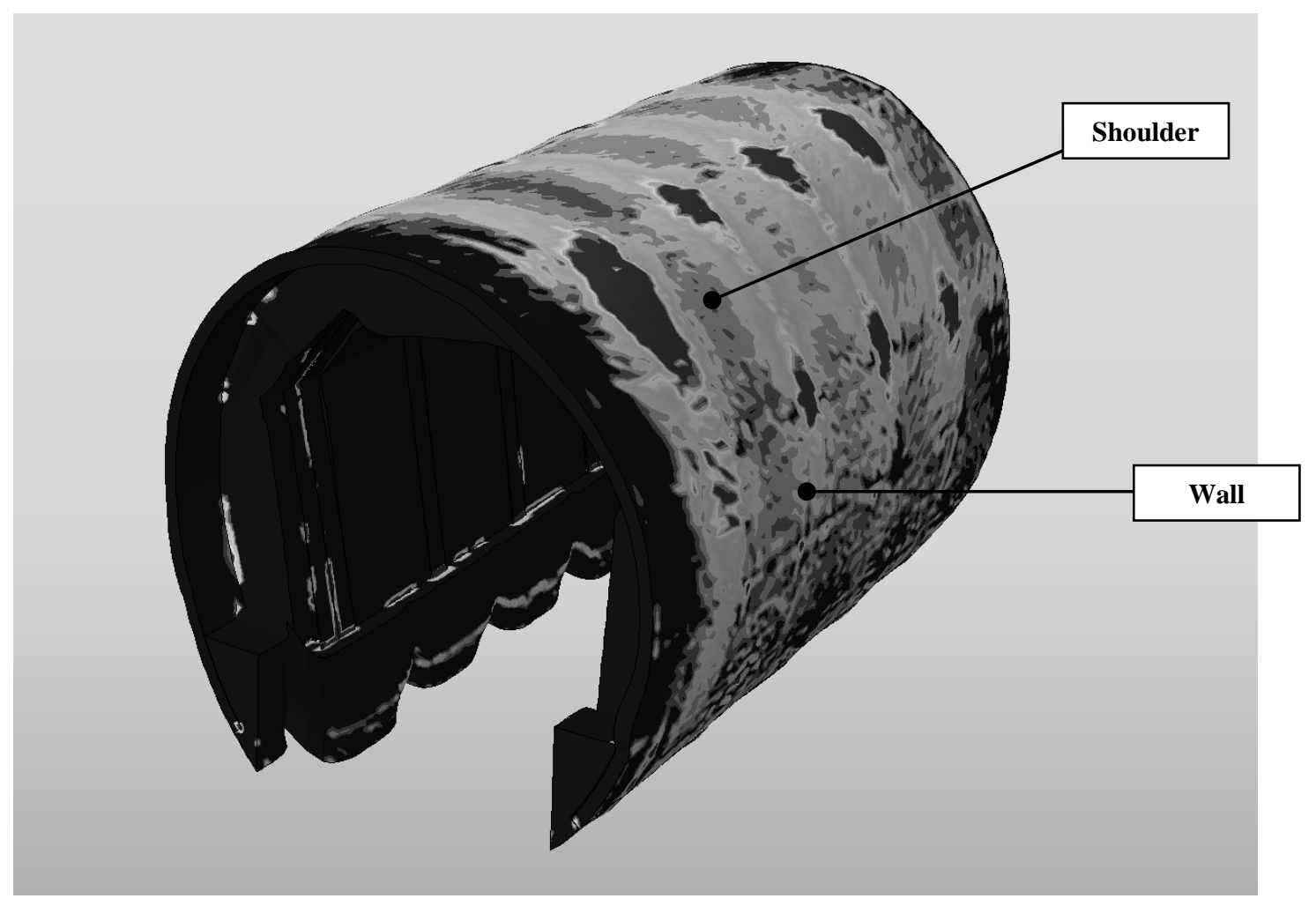

(i)

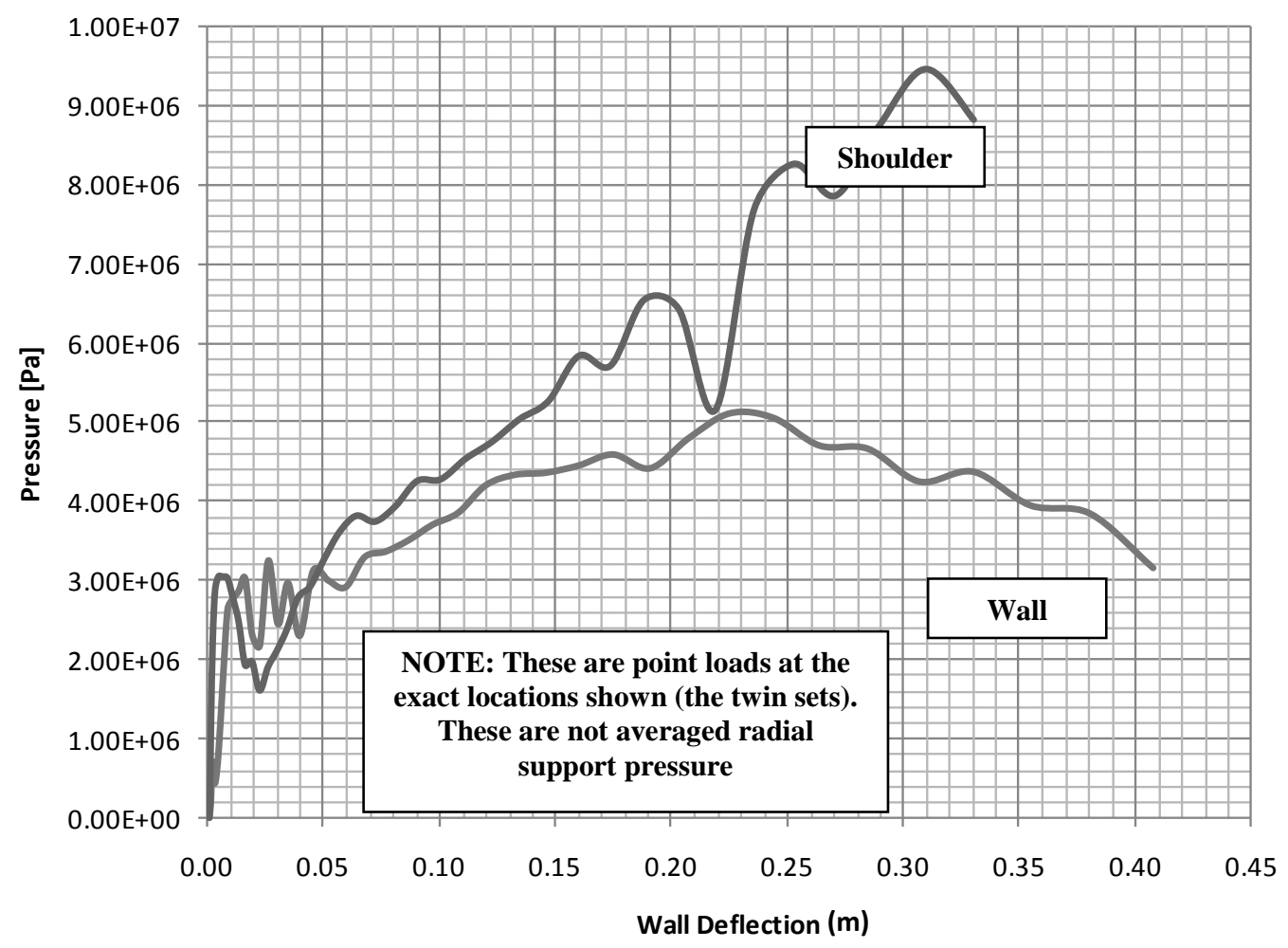

(ii)

Figure 7 Simulated point loads versus radial deflection for the steel set design. These are not the average radial support pressures 


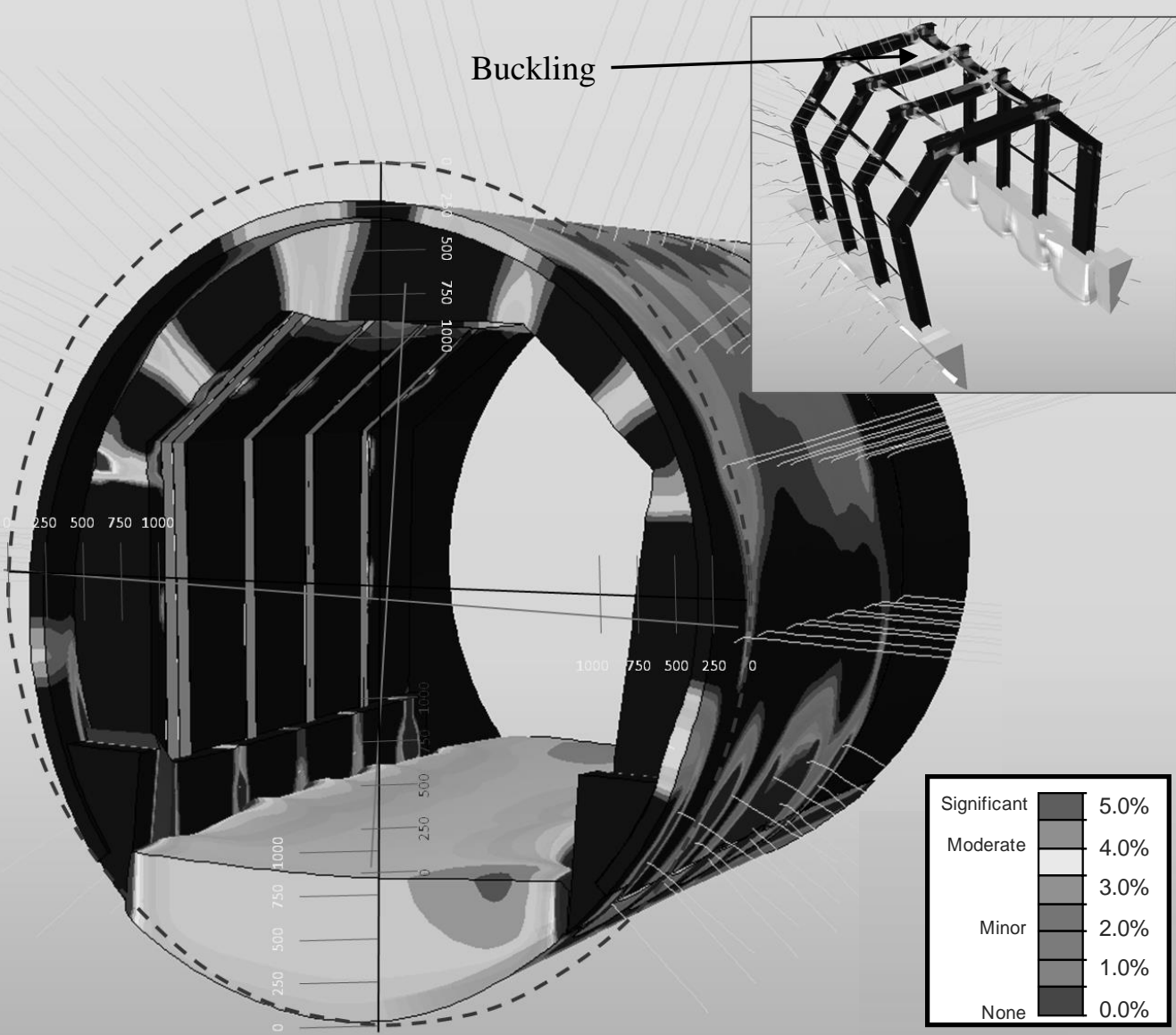

Figure 8 Simulated damage to steel set support at $325 \mathbf{~ m m}$ of closure

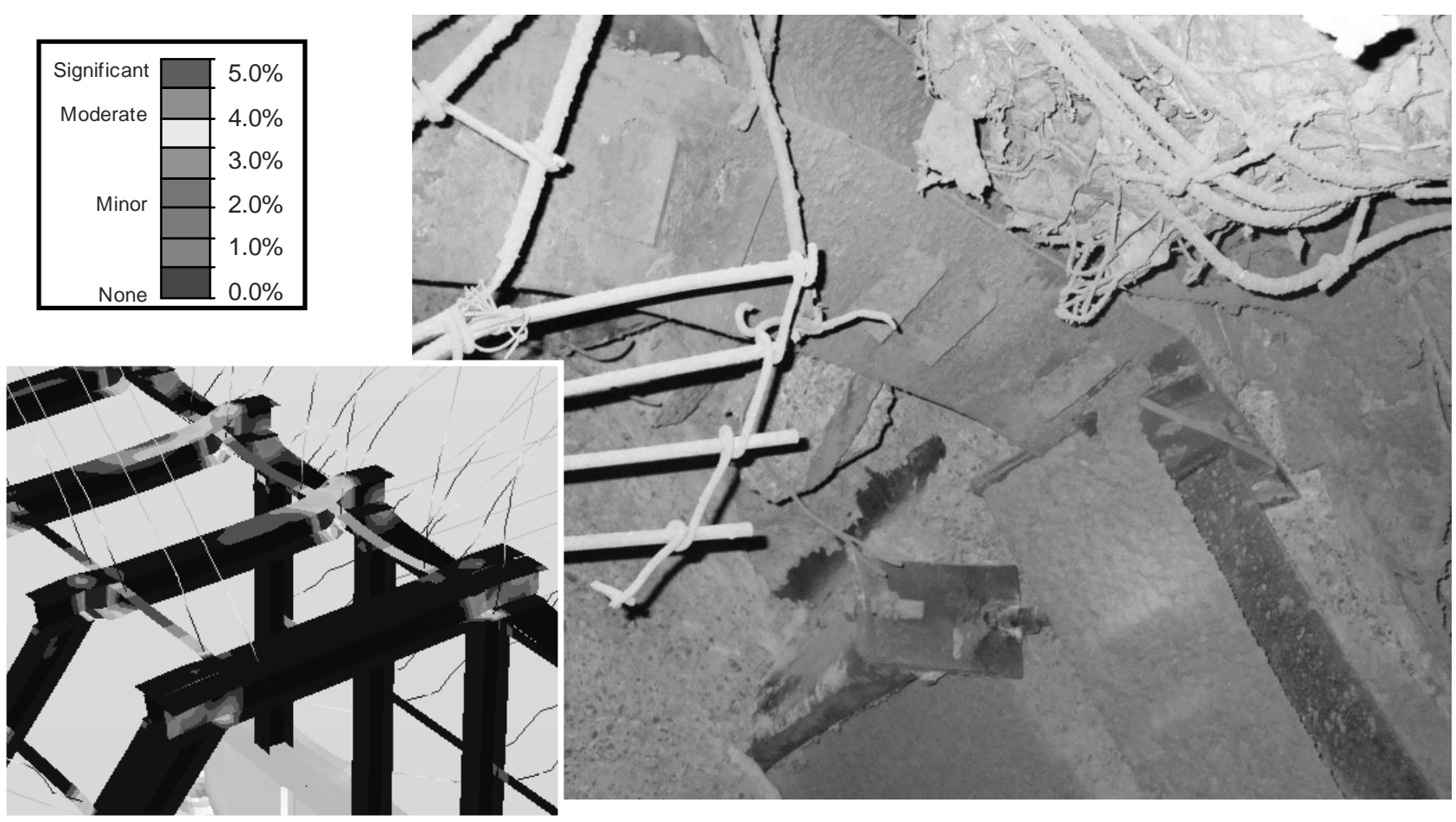

Figure 9 Example of steel set failure, and a close view of steel set failure in the model 

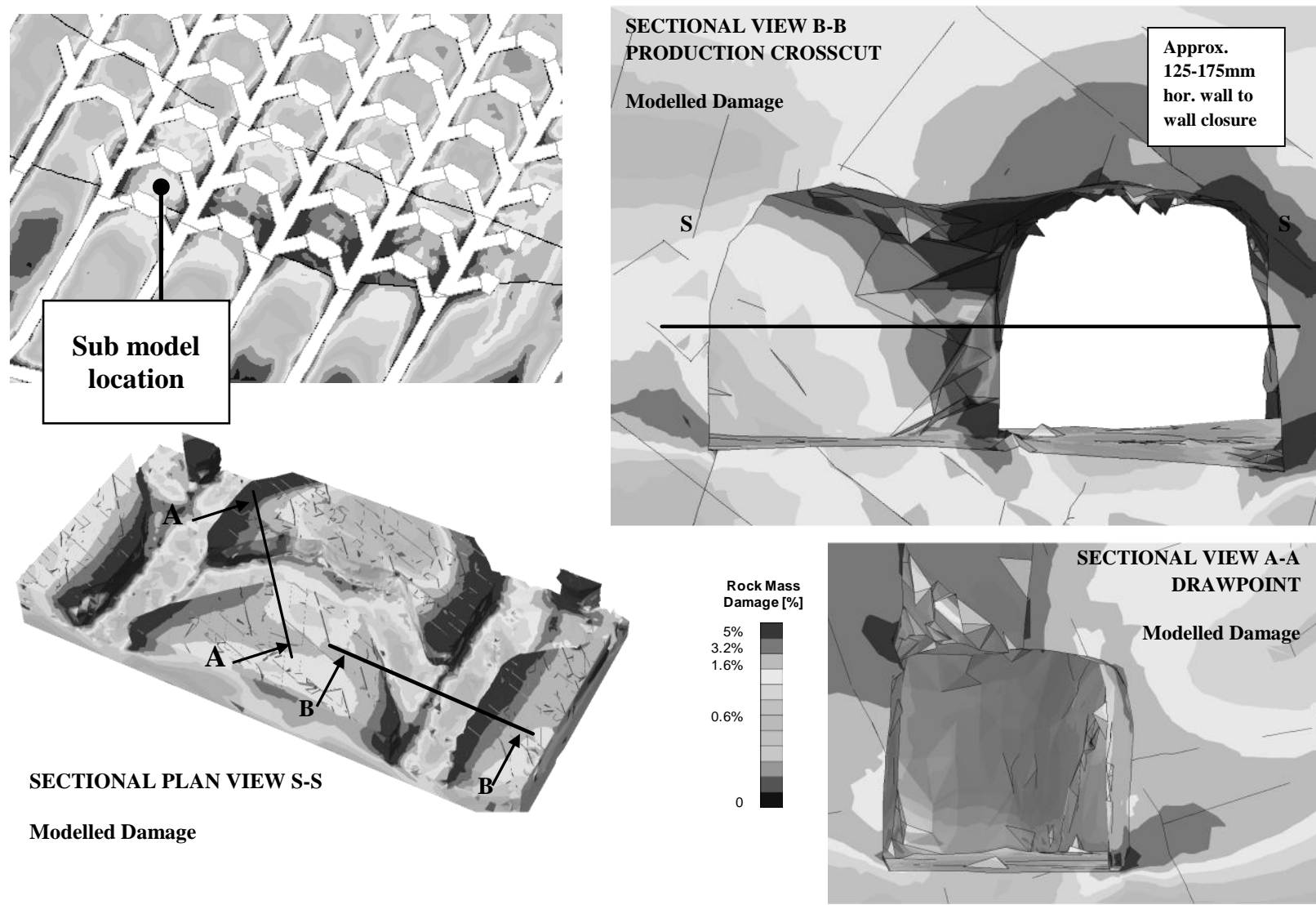

Modelled Damage

(a)
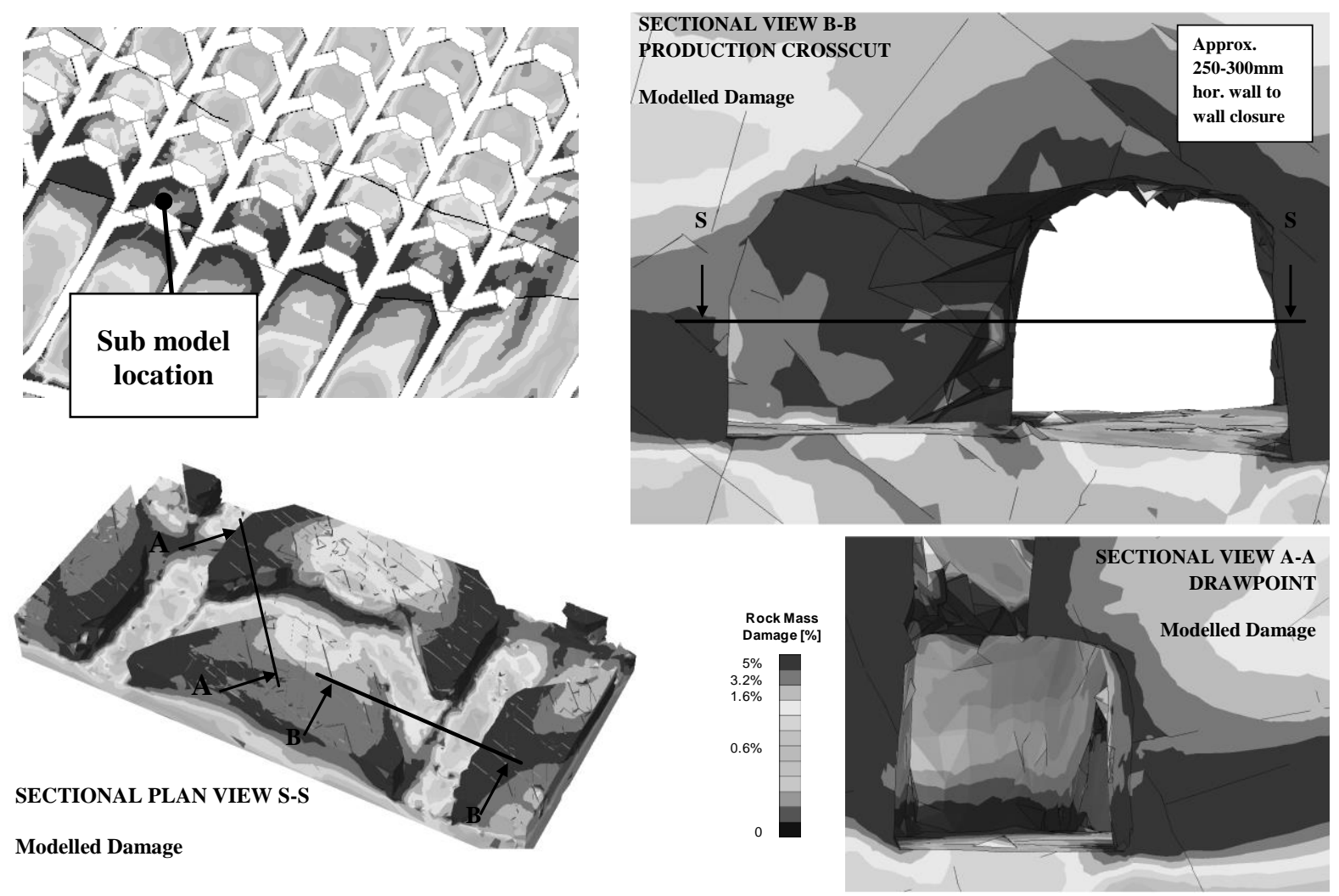

(b)

Figure 10 Simulated pillar rock mass damage at selected stages of extraction in an example 1/10 mine scale FE DFN model 


\section{Conclusions}

A number of different modelling concepts have been brought together in order to simulate support capacity and demand with a very high level of similitude. The intent is to capture the mechanisms of discontinuous deformation around complex geometries with high confidence. The magnitudes and extent of the deformation in the example cases was calibrated using field data, so show that realistic modelling of support is possible.

Common to the largest model geometries discussed here are well featured constitutive models, higher order elements and fine meshes, small excavation steps and appropriate boundary conditions. Modern non-linear, strain softening, dilatancy model packages allow for computation of models such as these in sufficiently short time frames and at a cost that makes the analysis feasible.

\section{References}

Beck, D.A. (2008) Multi-scale, non-linear numerical analysis of mining induced deformation, in Proceedings 42nd US Rock Mechanics Symposium and 2nd US-Canada Rock Mechanics Symposium, held in San Francisco, June 29 - July 2, 2008, ARMA, American Rock Mechanics Association.

Beck, D.A. and Reusch, F. (2009) A numerical investigation of scale effects on the behaviour of discontinuous rock, in Proceedings 43rd US Rock Mechanics Symposium held in Asheville, 2008, ARMA, American Rock Mechanics Association.

Davis, E.H. (1968) Theories of plasticity and the failure of soil masses, in Soil Mechanics: Selected Topics, Butterworths, London.

Drucker, D.C., Prager, W. and Greenberg, H.J. (1952) Extended limit design theorems for continuous media, Quarterly of Applied Mathematics, Vol. 9, pp. 381-389.

Hill, R. (1951) On the state of stress in a plastic-rigid body at the yield point, Philosophical Magazine, Vol. 42, pp. 868-875.

Hodge, P.G. (1958) The mathematical theory of plasticity, in Elasticity and plasticity, John Wiley and Sons, New York.

Lubliner, J. (1990) Plasticity Theory, Macmillan Publishing Company, New York. 
